\title{
Single Item Measure of Social Supports: Evaluation of construct validity during pregnancy
}

\section{Abstract}

Background: Lack of social support during pregnancy is associated with psychosocial vulnerability. The Single Item Measure of Social Supports (SIMSS) is included in a core outcome set to facilitate case-adjustment based on social support. Validity of the SIMSS has not been evaluated in childbearing women. We aimed to evaluate the construct validity of the SIMSS during pregnancy. Secondary aim was to evaluate an alternative short-measure of social support. Methods: Recruited women $(n=309)$ attending antenatal care were invited to complete the SIMSS, Multidimensional Scale of Perceived Social Support (MSPSS), Antenatal Risk Questionnaire (ANRQ), and Edinburgh Postnatal Depression Scale (EPDS). Convergent validity of SIMSS with MSPSS and hypothesis testing for psychosocial risk and depressive symptoms were conducted.

Results: Correlations were moderate between the SIMSS and MSPSS $\left(r_{s}=.35\right)$, but weak between the SIMSS and EPDS ( $\left.r_{s}=-.18\right)$; and SIMSS and ANRQ $\left(r_{s}=-\right.$ .22). In contrast, correlations were moderate between the MSPSS and EPDS ( $r_{s}=$ -.39) and ANRQ $\left(r_{s}=-.45\right)$. A shortened 3-item version of the MSPSS showed good psychometric properties and internal consistency reliability $(\mathrm{a}=.86)$.

Limitations: Findings relate to one Australian birthing sample during pregnancy. Replication of this study in larger, diverse maternity populations, including postpartum is recommended.

Conclusions: The SIMSS is a poor measure of social support during pregnancy with poor predictive ability to detect maternal vulnerability including depression and psychosocial risk. A revised 3-item version of the MSPSS was found to be a valid and reliable measure of social support.

Keywords: Patient reported outcome measure (PROM); pregnancy; Single Item Measure of Social Supports (SIMSS); core outcome set; validation study 


\section{Introduction}

Adequate social support during pregnancy optimises health in mothers and babies. Defined as any process through which social relationships might promote health and well-being (Cohen et al., 2000), social support operates by having both a direct (Broadhead et al., 1983), and buffering effect (Cohen and McKay, 1984). While strong correlations between social support, and psychosocial vulnerability (Austin et al., 2017 ), including maternal depression (Biaggi et al., 2015; Lancaster et al., 2010) are well established, the causal relationship is less clear. Psychosocial vulnerability might include a history of poor mental health, physical, sexual or emotional abuse, lack of support of support, poor relationship with mother or partner and stressors in the past year (Austin et al., 2013 ${ }^{\mathrm{a}}$ ). The short and long-term health-effects of maternal psychosocial vulnerability on babies is significant and includes prematurity, small for gestational age, and death before or within the first month of birth (Australian Institute of Health and Welfare [AIHW], 2018). Further, poor social support and maternal mental health adversely affect mother-infant relationships and child development (Balaji et al., 2007; Burchinal et al., 1996). As a potentially modifiable risk-factor, social support is an important construct to evaluate during pregnancy to inform the provision of high-quality maternity care.

\section{Measuring social support during pregnancy}

Recognising the significant impact of social support on maternal and newborn health, the International Consortium for Health Outcomes Measurement (ICHOM) included the Single Item Measure of Social Supports (SIMSS) (Blake and McKay, 1986) as a casemix outcome variable in their recently published core outcome set to measure value in maternity care (ICHOM, 2016; Nijagal et al., 2018).

The SIMSS asks, "How many people do you have near that you can readily count on for real help in times of trouble or difficulty, such as watch over children or pets, give ride to hospital or store, or help if you are sick?". 
To measure value in maternity care, all measures included in the ICHOM core outcome set must be psychometrically robust to produce valid and reliable data against which to benchmark care and outcomes. Other than one report of concurrent validity (Blake and McKay, 1986), there is little evidence regarding the psychometric properties of the SIMSS. Accordingly, there is an urgent need to evaluate the validity of the SIMSS to inform both feasibility and applicability of the measure within the core outcome set.

To facilitate the psychometric evaluation of the SIMSS a comparison measure was needed. A review of the literature identified the Multidimensional Scale of Perceived Social Support (MSPSS) to be widely used across different populations (Hardan-Khalil and Mayo, 2015). The reliability and validity of the MSPSS in the context of pregnancy was established in one study (Zimet et al., 1990) but further testing is also warranted. To test convergent and predictive validity of the SIMSS, validated measures of depression and psychosocial risk were needed. The Edinburgh Postnatal Depression scale is the most widely used, evaluated measure of symptoms of perinatal depression (Gibson et al., 2009) and is included in the core outcome set (ICHOM, 2016; Nijagal et al., 2018). However, the core set does not contain a measure of psychosocial risk, and the latest Australian Perinatal Mental Health Guidelines (where the current study is conducted) recommends use of the Antenatal Risk Questionnaire (ANRQ) (Austin et al, 2017 ) as a psychosocial screening tool. The technical performance of the ANRQ has been evaluated in pregnancy, is freely available, takes five minutes to complete and can be included in routine antenatal care (Austin et al., 2013; Reilly et al., 2015). In a review of perinatal psychosocial assessment tools, the ANRQ recorded acceptable performance, moderate validity, reliability and applicability, and performed higher than two other instruments in terms of ease of administration, acceptability, effectiveness, and implementability (Austin et al., 2017 ${ }^{\mathrm{b}}$ ). To inform the evidence-based inclusion of the SIMSS in the ICHOM core outcome set, the primary aim of this study was to evaluate construct, 
convergent and predictive validity of the SIMSS during pregnancy. A secondary aim was to evaluate an alternative short-measure of social support.

\section{Material and methods}

\subsection{Design, setting, participants}

The current study is part of a larger project described elsewhere (Slavin et al., 2019). A prospective, longitudinal, cohort study was conducted at one large birthing facility in south east Queensland with over 5100 births in 2018. Recruitment occurred between August 2017 and April 2018. Eligible participants were: (i) English-speaking; (ii) aged at least 18 years; (iii) 27-weeks gestation or less; and (iv) with access to email and mobile phone. Women under the current care of a psychiatrist were excluded. Data were collected at five time points, twice during pregnancy (baseline at enrolment and 36 weeks) and postpartum (week of birth, 6-, and 26- weeks). The current study reports baseline data only. Reporting of this study is guided by the STROBE statement for cohort studies. Participant recruitment is shown in Figure 1. 


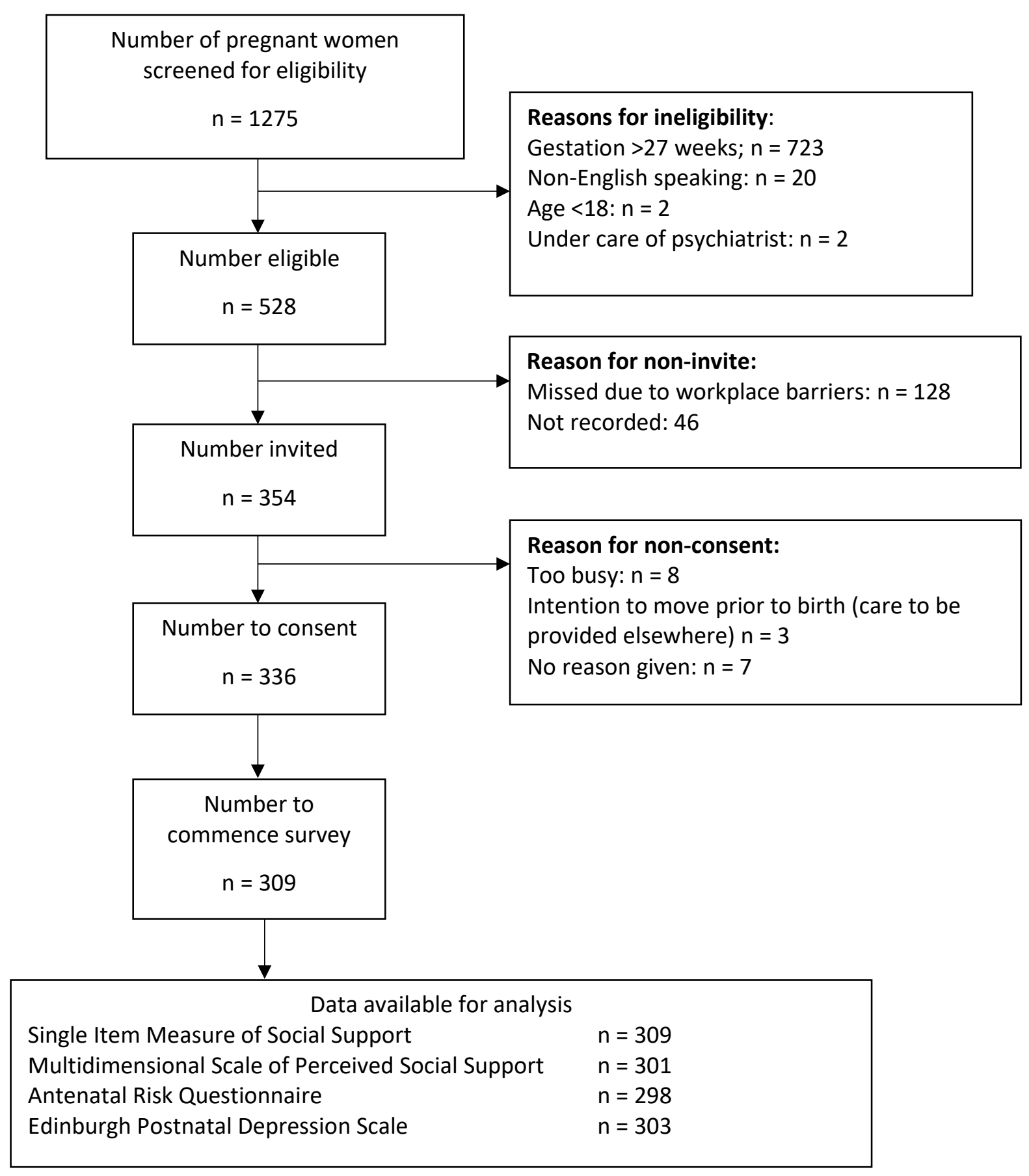

Figure 1 Flow diagram of participant recruitment and instrument completion

\subsection{Procedure}

Ethical approval was obtained to conduct this study from the participating Hospital and Health Service Human Research Ethics Committee (HREC/17/QGC/127) and University (GU Ref No: 2017/625). Eligible women attending antenatal care were approached, informed of the study aims, and provided with a detailed participant information booklet. Consenting 
women were administered a survey link by email and text message on the day of recruitment. Women who failed to respond were sent two friendly reminders and one telephone follow-up call, two-three days apart. Women who failed to respond to the first survey before 28 weeks gestation were deemed lost to follow-up. Women received no incentives for participation.

\subsection{Measures}

Baseline sociodemographic data included age and gestation.

\subsubsection{Single Item Measure of Social Supports}

The Single Item Measure of Social Supports (SIMSS) measures perceived availability of tangible (instrumental) social support network, by asking, "How many people do you have near that you can readily count on for real help in times of trouble or difficulty, such as watch over children or pets, give a ride to hospital or store, or help if you are sick?". Response options were $0,1,2$ to 5 , or 6 to 10 or more. A response of 0 or 1 indicates low tangible assistance; responses of 2 or more indicate high tangible assistance (Blake and McKay, 1986).

\subsubsection{Multidimensional Scale of Perceived Social Support}

The 12-item Multidimensional Scale of Perceived Social Support (MSPSS) is a self-report measure of both structural and functional perceived support from family (I get the emotional help and support I need from my family), friends (I can talk about my problems with my friends) and significant other (There is a special person with whom I can share my joys and sorrows $)$ scored on a 7-point Likert scale $(1=$ very strongly disagree to $7=$ very strongly agree) (Zimet et al., 1988). Scores range from 12 to 84 for a total score or computed for fouritem subscales of Family (FAM), Friends (FRI), and Significant Other (SO). The MSPSS has demonstrated strong internal consistency during pregnancy; $\alpha=.92$ (Total), .90 (Family), .94 (Friends), and .90 (Significant Other) in a United States sample of 265 pregnant women in 
their third trimester of pregnancy (Zimet et al., 1990) and has been translated and validated in 22 different languages (Dambi et al., 2018).

\subsubsection{The Edinburgh Postnatal Depression Scale (EPDS)}

The Edinburgh Postnatal Depression Scale is the most widely used (Gaynes et al., 2005) screening tool of depression symptoms during pregnancy (Murray and Cox, 1990).

Comprising 10 items on a 4-point Likert scale (score 0-3) women respond to statements relating to feelings of guilt, low energy, anhedonia, sleep disturbance and suicidal thoughts. Total scores range from $0-30$; higher scores indicate higher frequency or severity of symptoms. At the recommended cut-point of 13 or more to indicate 'at least probable minor depression' (Matthey et al., 2006) the EPDS has demonstrated 0.64 sensitivity, 0.90 specificity, and a positive predictive value of 0.50 (Murray and Cox, 1990).

\subsubsection{Antenatal Risk Questionnaire (ANRQ)}

The Antenatal Risk Questionnaire (ANRQ) is a 12-item self-report measure of psychosocial risk. Domains include mental health history, history of physical, sexual or emotional abuse or neglect, level of practical and emotional support from the partner, anxiety and perfectionism traits and stressors during the past 12 months (Austin et al., 2013). Five dichotomous items are scored as 'No' $(0)$ or 'Yes' (5). Seven items are scored from 0-5. Scores range from $5-$ 60 with higher scores indicative of greater psychosocial risk. A score of 23 or more is considered clinically significant as a predictor for postpartum depression with acceptable sensitivity (0.62), specificity (0.64), and a positive predictive value of 0.3 in a sample of 1196 Australian women (Austin et al., 2013).

\subsection{Approach to analysis}

All statistical analyses were conducted using the Statistical Package for Social Sciences (SPSS) for Windows version 25 (IBM Corp, 2017). Descriptive statistics describe the sample 
during pregnancy. Non-parametric statistics (median and interquartile range) are reported for all scales due to violations of the assumptions of normality. Missing data were managed using listwise deletion for computing total scale scores and Cronbach's $\alpha$, and pairwise deletion for all other analysis. Responses on the SIMSS were dichotomised into low support (score $0-1)$ and high support (score 2 or more) (Blake \& McKay 1986). EPDS scores were transformed into a dichotomous variable representing no probable minor depression (EPDS score 0 - 12) and probable minor depression (EPDS score 13 or more) (Matthey et al., 2006). ANRQ scores were transformed into a dichotomous variable representing low psychosocial risk (score 0-22) and high psychosocial risk (score 23 or more) to reflect the cut-off scores (Austin et al., 2013). All data manipulations are fully described within the accompanying tables. Incidence of low practical support (as measured by the SIMSS) is reported with $95 \%$ confidence interval (Clopper-Pearson exact). Statistical significance was taken as $p \leq .05$ throughout.

\subsubsection{SIMSS - Construct validity}

Construct validity refers to the measurement property used to evaluate the validity of the SIMSS for use in clinical practice and includes hypothesis testing (Mokkink et al., 2010). We further sub-classified hypothesis testing to include convergent and predictive validity. Evidence by Lancaster and colleagues (2010) were followed regarding the strength and direction of the expected relationships between social support and depression.

\subsubsection{Convergent validity}

Convergent validity was evaluated using Spearman Rank Order Correlation and interpreted using Cohen's criteria (Cohen, 1988). Measuring the construct of social support, a strong correlation was expected between the SIMSS and MSPSS. An inverse moderate correlation was expected between measures of social support and depression symptoms (EPDS) and psychosocial risk (ANRQ). 


\subsubsection{Predictive validity}

Receiver Operator Characteristics (ROC) were conducted to determine the overall precision of the SIMSS (ordinal score) and MSPSS (total score) in predicting depressive symptoms (according to the EPDS) and interpreted according to the criteria by Tape (no date). It was anticipated that the two social support measures would have similar predictive ability.

\subsubsection{Evaluation of an alternative short measure}

Two shortened versions of the MSPSS were also evaluated as alternative brief measures of social support. Firstly, the structural validity of the full 12-item MSPSS was explored using exploratory factor analysis. Principal components analysis (PCA) was used to extract the factors followed by oblique rotation of factors using Oblimin rotation $($ delta $=0)$. The number of factors to be retained was guided by three decision rules: Kaiser's criterion (eigenvalues above 1), inspection of the scree plot, and Horn's parallel analysis (Horn, 1965). The eigenvalues obtained from PCA were compared with those from a randomly generated dataset of the same size. Only eigenvalues exceeding the values obtained from the corresponding random data set were retained for further investigation. Parallel analysis was conducted using the software developed by Watkins (2000).

Using the results obtained from the full 12-item evaluation, the top two loading items and top single loading item from each of the three subscales were then evaluated as 6-item and 3-item scales. Internal consistency reliability was assessed using Cronbach's alpha coefficient $(\alpha)$ and interpreted according to the criteria of Nunnally (Nunnally, 1978). Correlations between both shortened versions and the EPDS and ANRQ were subsequently conducted using Spearman Rank Order Correlation. 


\section{Results}

\subsection{Sample characteristics}

Table 1 presents the demographic and psychosocial characteristics of 309 pregnant participants. The SIMSS was completed by all and the MSPSS was completed by 301 (97.4\%) participants.

[Insert Table 1 about here]

\subsection{Single Item Measure of Social Support}

Incidence of low practical support

Nine $(2.9 \%)$ women reported having no-one they could rely on in times of need, $40(12.9 \%)$ women reported having one person, $213(68.9 \%)$ reported having between 2 and 5 people and $47(15.2 \%)$ women reported having 6 or more people. Low practical support (defined as 0 or 1 person) was thus reported by 49 (15.9\%; 95\% CI: 12.0-20.4) women.

\subsection{Correlation between SIMSS and MSPSS}

A moderate correlation between the MSPSS (total score) and the SIMSS was found, explaining $12 \%$ of the variance $\left(r_{s}(301)=.35, p=<.001\right)$ (see Table 2$)$.

[Insert Table 2 about here]

\subsection{Correlation between social support and psychosocial risk}

The SIMSS was weakly and negatively correlated with both depressive symptoms $\left(r_{s}=-.18\right)$ and psychosocial risk $\left(r_{s}=-.22\right)$ explaining $3 \%$ and $4.5 \%$ of the variance respectively. The MSPSS was moderately and negatively correlated to depressive symptoms $\left(r_{s}=-.39\right)$ and psychosocial risk $\left(r_{s}=-.43\right)$, explaining $15.2 \%$ and $18.5 \%$ of the variance (Table 2$)$.

\subsection{Social support as a predictor of depressive symptoms}

ROC curve analysis demonstrated the SIMSS to have poor precision in categorising women reporting depressive symptoms $(\mathrm{AUC}=0.68: 95 \% \mathrm{CI}$ : 0.53 - 0.83; Standard Error $(\mathrm{SE})=.08$, 
$p=.03)$ while the MSPSS had good precision (AUC $=0.83,95 \%$ CI: $0.73-0.92$, SE .05 , $p<.001)$. Of the 303 women with complete data for both the SIMSS and EPDS, 5 of the 12 women above the threshold for at least probable minor depression reported low tangible support (SIMSS) and 7 reported high tangible support. A further 43 women who reported low tangible support were below the threshold for probable depression (sensitivity $=0.42$; specificity $=0.85)$.

\subsection{Evaluation of the MSPSS}

\subsubsection{Structural validity: 12-item MSPSS}

The internal structure of the 12-item MSPSS was explored using Principal Components Analysis (PCA). Inspection of the correlation matrix revealed all coefficients at .3 and above. The Kaiser-Meyer-Olkin value was .9 (Kaiser, 1970) and Bartlett's Test of Sphericity (Bartlett, 1954) reached statistical significance, supporting the factorability of the correlation matrix. PCA revealed three components with eigenvalues exceeding 1, explaining $75.0 \%$, $9.3 \%$ and $5.7 \%$ of the variance. An inspection of the respective screeplots revealed three components, supporting a three-factor solution. Using Catell's (1966) scree test, it was decided to retain three components. Results of the Parallel Analysis however, showed only one component with an eigenvalue exceeding the corresponding criterion values for a randomly generated data matrix of the same size, (12 variables, 309 participants, 100 replications).

The three-component solution explained $90.0 \%$ of the variance. To aid in the interpretation of these three components, Oblimin rotation was performed. Item loadings for each of the 12 statements are shown in Table 5 (loadings of .3 or above are bolded). Consistent with the findings of Zimet et al (1988; 1990), items 1, 2, 5 and 10 loaded on the first component (Significant Other); items 6, 7, 9 and 12 loaded on the second component (Friends); and items 3, 4, 8 and 11 loaded on a third component (Family) (Table 3). 
Inspection of the component correlation matrix revealed strong intercorrelations between the three components. The correlation between Significant Other and Friends factors was $r=.66$. Correlations between Family and Significant Other was $r=.77$ and between Family and Friends was $r=.70$. Strong internal consistency reliability was found for the total MSPSS scale $(\alpha=.97)$; Significant Other $(\alpha=.96)$, Family $(\alpha=.95)$ and Friends $(\alpha=.97)$ subscales.

[Insert Table 3 about here]

\subsubsection{Internal consistency and construct validity of two brief versions of social support}

A 6-item and 3-item version of the MSPSS were subsequently evaluated. The 6-item version (MSPSS-R-6) comprises the strongest two items of each components (Significant Other: items 2 and 1; Friends: items 7 and 12; Family: items 4 and 3). The 3-item version (MSPSS-R-3) comprises of the strongest single item of each component (Significant Other: item 2; Friends: item 7; Family: item 4). Internal consistency reliability was good for the MSPSS-R-6 $(\alpha=.93)$ and MSPSS-R-3 $(\alpha=.86)$. Total scores for the MSPSS-R-6 and MSPSS-R-3 were $M d n=39$, $I Q R=7$, range 6-42; and $M d n 19, I Q R=4$, range 3-21, respectively. Correlations between the MSPSS (total score) and MSPSS-R-6 $\left(r_{s}(301)=.99, p<.001\right)$ and MSPSS-R-3 scales $\left(r_{s}\right.$ $(301)=.97, p<.001)$ explained $98 \%$ and $94 \%$ of variance respectively. A moderate negative correlation was seen between the EPDS total score and MSPSS-R-6 $\left(r_{s}(301)=-.39, p<.001\right)$ and MSPSS-R-3 $\left(r_{s}(301)=-.39, p<.001\right)$ scales explaining $15.2 \%$ of the variance; and a moderate negative correlation between the ANRQ and MSPSS-R-6 $\left(r_{s}(301)=-.43, p=\right.$ $<.001)$ and MSPSS-R-3 $\left(r_{s}(301)=-.45, p<.001\right)$ tools, explaining $18.5 \%$ and $20.3 \%$ of the variance respectively.

\section{Discussion}

This study evaluated the construct, convergent and predictive validity of the Single Item Measure of Social Supports to inform its inclusion in the ICHOM core outcome set for 
pregnancy and childbirth. While the SIMSS revealed a moderate correlation with another validated measure of social support (MSPSS), the weak correlation observed between the SIMSS and depressive symptoms (EPDS) and psychosocial risk (ANRQ), indicated a lack of convergent validity. The poor predictive validity of the SIMSS to detect depressive symptoms as indicated by ROC analysis, further confirmed this finding. As a measure of social support, the SIMSS was thus found to lack adequate construct validity. In contrast, the 12-item MSPSS revealed good structural validity, internal consistency reliability and adequate construct validity, demonstrated by moderate correlations with depressive symptoms and psychosocial risk and good predictive ability for at least probable minor depression. Further, two shortened versions of the MSPSS were found to be have similar robust psychometric properties.

For childbearing women, inadequate social support is consistently associated with psychosocial vulnerability (Knight et al., 2018), including depression (Biaggi et al., 2015; Lancaster et al., 2010) and contributes to significant maternal morbidity and mortality (Knight et al., 2018). In this context, the SIMSS was an inadequate predictor of maternal morbidity. Current evidence regarding the psychometric properties of the SIMSS is however sparse, limiting comparison. The SIMSS was originally described three decades ago as a predictor of morbidity (1986), including disability days or restriction in usual activity due to illness or injury (United States Department of Health, 1978). Using a sample of 480 men (40\%) and women (60\%) aged between 20 and 65 years, Blake and McKay (1986) evaluated concurrent validity against a '12-item general social support index'. Even though the authors referred to results of another paper (McKay et al., 1985), the lack of item descriptions and psychometric data limits the conclusions that can be drawn regarding the robustness of the SIMSS. Similarly, while the SIMSS has been reported, in mixed-gender patients with rheumatoid arthritis (Caplan et al., 2014), multiple sclerosis (Weiland et al., 2015), ethnically 
diverse older women (Laganà et al., 2011) and women with breast cancer (Turner-Cobb et al., 2004), psychometric analysis is not generally reported.

\subsection{Theoretical interpretation of findings}

Social support represents a complexity of inter-related dimensions incorporating both structural and functional (Leahy-Warren et al., 2012; Nausheen et al., 2009) components. As a numerical measure of only one dimension of functional support (instrumental) the SIMSS fails to consider the source or quality of support in terms of emotional, informational or social companionship. To illustrate its limitations, a pregnant woman may perceive she has available support of several people, but the support may only be in practical (instrumental) terms. On the other hand, a pregnant woman may perceive she is supported by only one person who meets all her emotional, informational, instrumental and social companionship needs. Effects of the missing dimensions become even clearer when considered in the association with depressive symptoms. The low correlation between the single item measure and high EPDS scores $\left(r_{s}=-.18\right)$ with accompanying low sensitivity $(0.42)$ and high specificity (0.85) suggests a woman can have at least probable minor depression even if she perceives practical support from numerous people. Similarly, some women with low tangible social support may not be at risk of probable depression. It appears that counting sources of support is not a useful measure of social support. As Nausheen et al (2009) points out, it is critical to distinguish network size from the source, as network size is not an indicator of quality or adequacy of support and may not benefit health outcomes. It is important to note, that while the SIMSS lacked construct validity as a numerical measure of support, it is possible that pregnant women who lack tangible social support ( 0 or 1 person) may represent a particularly vulnerable group of women. More research in this area is warranted. 


\subsection{Multidimensional Scale of Perceived Social Support- A robust measure of social support}

The MSPSS accounted for both structural and functional components of support. Although the MSPSS has been validated during pregnancy, psychometric evaluation in Australian maternity populations is limited and further psychometric evaluation was warranted. In line with others (Zimet et al., 1988; 1990), the current findings confirm the MSPSS to be a robust measure of social support. Congruent with Zimet and colleagues (1990) Principal Component Analysis of the 12-item MSPSS supported a three-factor structure during pregnancy. Itemloadings on the three subscales ranged from .86 to .94 which were slightly higher than that of Zimet who reported loadings between .82 and .89 . This may be explained by differences in timing of data collection (Hetherington et al., 2019). Zimet et al (1990) collected data from 265 pregnant women in their third trimester while the current study collected data from pregnant women in the first and second trimesters when women are likely to be in paid employment. Consistent with Zimet et al (1990) strong internal consistency reliability ( $\alpha=$ $\geq .90$ to .97 ), was recorded during pregnancy exceeding the recommended maximum of .90 recommended by Streiner (2003). This finding indicated possible item redundancies for this population supporting the evaluation of a shorter instrument.

Subsequent analysis of two shortened versions of the MSPSS (6- and 3-items) revealed strong internal consistency reliability $(\mathrm{a}=.93, .86)$, strong correlations with the 12 -item MSPSS $\left(r_{s}=.99, .97\right)$, and moderate inverse correlations with depressive symptoms $\left(r_{s}=-.39\right.$, $-.39)$ and psychosocial vulnerability $\left(r_{s}=-.43,-.45\right)$. The 3 -item version of the MSPSS, which includes item 2 (There is a special person with whom I can share my joys and sorrows), item 7 (I can count on my friends when things go wrong) and item 4 (I get the emotional help and support I need from my family), was found to be as valid and reliable as both the full 12-item and shortened 6-item versions of the tool. 


\subsection{Strengths and limitations}

The psychometric evaluation of two social support measures during pregnancy is a major strength of this study and informs evidence-based recommendations. Further, the inclusion of two recommended measures of depressive symptoms and psychosocial risk (Austin et al., 2017) make the findings particularly relevant in the Australian maternity care context. Our study does however have some limitations. Firstly, despite a review of the literature using several search terms including 'Single Item Measure of Social Support', 'SIMSS', 'single item', 'single instrument', 'single measure', 'brief measure', 'ultra-short measure' as well as a search using the full question, limited evidence was found regarding the psychometric properties of the SIMSS. It is possible, however, that as a single item measure, the question may have been used without citation and studies using the item may have been missed. Further we measured depressive symptoms in one Australian sample, at one time point, using a screening method rather than formal diagnostic criteria. While formal diagnosis is a more robust measure of depression, the method used in the current study is in line with other studies and does lend itself to future meta-analysis (Lancaster et al., 2010). We measured perceived social support at one time point and scale stability was not evaluated. The trajectory of social support during the peripartum period is reported to be relatively stable (Hetherington et al., 2019) therefore our findings should not be significantly impacted by our timing of measurement. Our sample size was calculated to meet our broader study aims and the number of women who recorded a complete absence of social support was small $(n=9)$. While it is possible our findings may lack power and precision, the high number of statistically significant results do lend support to our findings. Future research in larger and more diverse maternity populations, and over different time points during pregnancy and postpartum will address these limitations. 


\subsection{Clinical and research implications}

Maternal vulnerability in terms of depressive symptoms, psychosocial risk are important indicators of health-related morbidity in mothers and their babies (Austin et al., 2017 ). Consistent with the literature, social support has been shown to be an important maternal outcome and a valuable inclusion the ICHOM core outcome set. Vulnerable women who lack adequate social support present a particularly at-risk group for a range of adverse outcomes. Further research is needed to determine how the needs of these women may differ to those of women with adequate social support. Screening and evaluation of social support to facilitate the implementation of early interventions during pregnancy are warranted but rely on the use of psychometrically robust measurement tools. Despite undergoing an in-depth development process by an international collaborative working party, the SIMSS was chosen as the outcome measure within the core outcome set, despite a lack of evidence regarding its psychometric properties. The outcome was included as a casemix variable to facilitate caseadjustment, a method that accounts for variation across cohorts in baseline patient factors, when comparing health outcomes. However, our findings show the SIMSS to be an inadequate measure of social support. In contrast, a shortened 3-item version of the MSPSS was shown to be a valid and reliable measure of social support and is suggested as an alternative measure in the ICHOM core outcome set for pregnancy and childbirth.

\subsection{Conclusions}

The SIMSS was found to lack adequate construct, convergent and predictive validity. Our findings do not support the inclusion of the SIMSS in the ICHOM core outcome set. A shortened 3-item version of the MSPSS was found it be a valid and reliable measure of social support. Inclusion of this short scale should not significantly impact survey length. 


\section{Acknowledgements}

We would like to acknowledge the ongoing support of the women who participated in this study. We would also like to acknowledge Dr Julie Pallant for her ongoing support and statistical guidance.

\section{Funding information}

The MoMeNT study was supported by a grant awarded by the Gold Coast Hospital and Health Service Research Grants Committee (Ref: 015-01.02.17). The funding body played no role in the study design, data collection, data analysis, or interpretation of findings.

\section{Ethics approval and consent to participate}

Ethical approval was obtained to conduct this study from Gold Coast Hospital and Health Service Human Research Ethics Committee (HREC/17/ QGC/127) and Griffith University (GU Ref No: 2017/625). Written informed consent to participate was obtained from all participants.

\section{Competing interests}

The authors declare that they have no competing interests. 


\section{References}

Australian Institute of Health and Welfare [AIHW]., 2018. Perinatal Deaths in Australia 2013-2014. Cat. No. PER 94. https://www.aihw.gov.au/getmedia/78784f2e-2f6147ea-9908-84b34441ae0a/aihw-per-94.pdf/ (accessed 10 August 2019).

Austin, M.-P., Colton, J., Priest, S., Reilly, N., \& Hadzi-Pavlovic, D., 2013. The Antenatal Risk Questionnaire (ANRQ): Acceptability and use for psychosocial risk assessment in the maternity setting. Women and Birth, 26(1), 17-25. https://doi:https://doi.org/10.1016/j.wombi.2011.06.002.

Austin, M.-P., Highet, N., \& Expert Working Group., 2017 . Mental Health Care in the Perinatal Period: Australian Clinical Practice Guideline. Centre of Perinatal Excellence, Melbourne. https://cope.org.au/wp-content/uploads/2017/10/Final-COPEPerinatal-Mental-Health-Guideline.pdf (accessed 10 September 2019).

Austin, M.-P., Highet, N., \& Expert Working Group., 2017 . Australian Perinatal Mental Health Guideline Evidence Review. Technical Report Part A Overall approach and governance. https://www.cope.org.au/wp-content/uploads/2017/06/01.-PARTA_GOVERNANCE_Technical-Report_DRAFT_31May17_circ.pdf (accessed 10 September 2019).

Balaji, A.B., Claussen, A.H., Smith, D.C., Visser, S.N., Morales, M.J., \& Perou, R., 2007. Social support networks and maternal mental health and well-being. Journal of Women's Health, 16(10), 1386-1396. https://doi:10.1089/jwh.2007.CDC10.

Bartlett, M., 1954. A note on the multiplying factors for various chi square approximations. Journal of the Royal Statistical Society, 16 (Series B), 296-298.

Biaggi, A., Conroy, S., Pawlby, S., \& Pariante, C.M., 2015. Identifying the women at risk of antenatal anxiety and depression: A systematic review. Journal of Affective Disorders, 191, 62-77. https://doi:10.1016/j.jad.2015.11.014.

Blake, R.L, Jr., \& McKay, D.A., 1986. A Single-Item Measure of Social Supports as a predictor of morbidity. The Journal of Family Practice, 22(1). 82-84.

Broadhead, W.E., Kaplan, B.H., James, S.A., Wagner, E.H., Schoenbach, V.J., Grimson, R., . . . Gehlbach, S.H., 1983. The epidemiologic evidence for a relationship between social support and health. Am J Epidemiol, 117(5), 521-537. https://doi:10.1093/oxfordjournals.aje.a113575.

Burchinal, M.R., Follmer, A., \& Bryant, D.M., 1996. The relations of maternal social support and family structure with maternal responsiveness and child outcomes among African 
American families. Developmental Psychology, 32(6), 1073-1083.

https://doi:10.1037/0012-1649.32.6.1073.

Caplan, L., Wolfe, F., Michaud, K., Quinzanos, I., \& Hirsh, J.M., 2014. Strong association of health literacy with functional status among rheumatoid arthritis patients: A crosssectional study. Arthritis Care \& Research, 66(4), 508-514. https://doi:10.1002/acr.22165.

Cattell, R.B., 1966. The Scree Test for the number of factors. Multivariate Behavioral Research, 1(2), 245-276. https://doi:10.1207/s15327906mbr0102_10.

Cohen, J.W., 1988. Statistical Power Analysis For The Behavorial Sciences, second ed. Erlbaum, New York.

Cohen, S., Gottlieb, B.H., Underwood, L.G., 2000. Social relationships and health, in: Cohen, S., Underwood, L.G., Gottlieb, B.H. (Eds.), Social Support Measurement and Intervention. A Guide for Health and Social Scientists., New York, pp. 3-28.

Cohen, S., McKay, G., 1984. Social support, stress and the buffering hypothesis: A theoretical analysis, in: Baum, A., Singer, J.E.,Taylor, S.E. (Eds.), Handbook of Psychology and Health, (Vol. 4)., Hillsdale, NJ, pp. 253-267.

Dambi, J.M., Corten, L., Chiwaridzo, M., Jack, H., Mlambo, T., \& Jelsma, J., 2018. A systematic review of the psychometric properties of the cross-cultural translations and adaptations of the Multidimensional Perceived Social Support Scale (MSPSS). Health and Quality of Life Outcomes, 16(1), 80. https://doi.org/10.1186/s12955-018-0912-0.

Gaynes, B., Gavin, N., Meltzer-Brody, S., Lohr, K., Swinson, T., Gartlehner, G., . . Miller, W., 2005. Perinatal Depression: Prevalence, Screening Accuracy, and Screening Outcomes. Evidence Report/Technology Assessment No. 119. AHRQ Publication No. 05-E006-2. https://www.ncbi.nlm.nih.gov/books/NBK11838/ (accessed 10 October 2019).

Gibson, J., McKenzie-McHarg, K., Shakespeare, J., Price, J., \& Gray, R., 2009. A systematic review of studies validating the Edinburgh Postnatal Depression Scale in antepartum and postpartum women. Acta Psychiatrica Scandinavica, 119(5):350-64.

Hardan-Khalil, K., \& Mayo, A.M., 2015. Psychometric properties of the Multidimensional Scale of Perceived Social Support. Clinical Nurse Specialist, 29(5), 258-261. https://doi:10.1097/NUR.0000000000000148. 
Hetherington, E., McDonald, S., Williamson, T., \& Tough, S., 2019. Trajectories of social support in pregnancy and early postpartum: findings from the All Our Families cohort. Social Psychiatry and Psychiatric Epidemiology. https://doi:10.1007/s00127019-01740-8.

Horn, J.L., 1965. A rationale and test for the number of factors in factor analysis. Psychometrika, 30(2):179-85.

IBM Corp. (2017). IBM SPSS Statistics for Windows, Version 25.0. Armonk, New York. International Consortium for Health Outcomes Measurement [ICHOM]., 2016. Pregnancy \& Childbirth. Data collection reference guide. Version 1.1. https://ichom.org/files/medical-conditions/pregnancy-and-childbirth/pregnancychildbirth-reference-guide.pdf (accessed 9 April 2019).

Kaiser, H.F., 1970. A second generation little jiffy. Psychometrika, 35(4), 401-415. https://doi:10.1007/bf02291817.

Knight, M., Bunch, K., Tuffnell, D., Jayakody, H., Shakespeare, J., Kotnis, R., . . on behalf of MBRRACE-UK., 2018. Saving Lives, Improving Mothers' Care - Lessons learned to inform maternity care from the UK and Ireland Confidential Enquiries into Maternal Deaths and Morbidity 2014-16. https://www.npeu.ox.ac.uk/downloads/files/mbrrace-uk/reports/ (accessed 11 August 2019).

Laganà, L., Bratly, M.L., \& Boutakidis, I., 2011. The validation of a new measure quantifying the social quality of life of ethnically diverse older women: Two crosssectional studies. BMC Geriatrics, 11(1), 60-60. https://doi:10.1186/1471-2318-11-60.

Lancaster, C.A., Gold, K.J., Flynn, H.A., Yoo, H., Marcus, S.M., \& Davis, M.M., 2010. Risk factors for depressive symptoms during pregnancy: a systematic review. American Journal of Obstetrics and Gynecology, 202(1), 5-14. https://doi:10.1016/j.ajog.2009.09.007.

Leahy-Warren, P., McCarthy, G., \& Corcoran, P., 2012. First-time mothers: social support, maternal parental self-efficacy and postnatal depression. Journal of Clinical Nursing, 21(3-4), 388-397. https://doi:10.1111/j.1365-2702.2011.03701.x.

Li, T., Guo, N., Jiang, H., Eldadah, M., \& Zhuang, W., 2019. Social support and second trimester depression. Midwifery, 69, 158-162. https://doi:10.1016/j.midw.2018.11.012.

Matthey, S., Henshaw, C., Elliott, S., \& Barnett, B., 2006. Variability in use of cut-off scores and formats on the Edinburgh Postnatal Depression Scale-implications for clinical 
and research practice. Archives of Women's Mental Health, 9(6), 309-315. https://doi $10.1007 / \mathrm{s} 00737-006-0152-\mathrm{x}$

McKay, D.A., Blake, R.L., Jr., Colwill, J.M., Brent, E.E., McCauley, J., Umlauf, R., . . . Kivlahan, D., 1985. Social supports and stress as predictors of illness. J Fam Pract, $20(6), 575-581$.

Mokkink, L.B., Terwee, C.B., Patrick, D.L., Alonso, J., Stratford, P.W., Knol, D.L., . . de Vet, H.C.W., 2010. The COSMIN study reached international consensus on taxonomy, terminology, and definitions of measurement properties for health-related patient-reported outcomes. Journal of Clinical Epidemiology, 63(7), 737-745. https://doi:10.1016/j.jclinepi.2010.02.006.

Murray, D., \& Cox, J.L., 1990. Screening for depression during pregnancy with the Edinburgh Depression Scale (EDDS). Journal of Reproductive and Infant Psychology, 8(2), 99-107. https://doi:10.1080/02646839008403615.

Nausheen, B., Gidron, Y., Peveler, R., \& Moss-Morris, R., 2009. Social support and cancer progression: A systematic review. Journal of Psychosomatic Research, 67(5), 403415. https://doi:10.1016/j.jpsychores.2008.12.012.

Nijagal, M.A., Wissig, S., Stowell, C., Olson, E., Amer-Wahlin, I., Bonsel, G., . . Franx, A., 2018. Standardized outcome measures for pregnancy and childbirth, an ICHOM proposal. BMC Health Services Research, 18(1), 953. https://doi:10.1186/s12913018-3732-3.

Nunnally, J.C., 1978. Psychometric Theory, fourth ed. McGraw-Hill, New York.

Racine, N., Madigan, S., Plamondon, A., Hetherington, E., McDonald, S., \& Tough, S., 2018.Maternal adverse childhood experiences and antepartum risks: the moderating role of social support. Archives of Women's Mental Health. 21(6), 663-670. https://doi:10.1007/s00737-018-0826-1.

Reilly, N., C. Yin, L. Monterosso, S. Bradshaw, K. Neale, B. Harrison \& M. P. Austin (2015). "Identifying psychosocial risk among mothers in an Australian private maternity setting: A pilot study." Aust N Z J Obstet Gynaecol 55(5): 453-458.

Slavin, V., Gamble, J., Creedy, D.K., Fenwick, J., \& Pallant, J., 2019. Measuring physical and mental health during pregnancy and postpartum in an Australian childbearing population. Validation of the PROMIS Global Short Form. BMC Pregnancy And Childbirth. 19, 370 https://doi:10.1186/s12884-019-2546-6. 
Stapleton, L.R.T., Schetter, C.D., Westling, E., Rini, C., Glynn, L.M., Hobel, C.J., \& Sandman, C.A., 2012. Perceived partner support in pregnancy predicts lower maternal and infant distress. Journal of Family Psychology, 26(3), 453-463. https://doi:10.1037/a0028332.

Streiner, D.L., 2003. Starting at the beginning: an introduction to coefficient alpha and internal consistency. Journal of Personality Assessment, 80(1), 99-103. https://doi:10.1207/S15327752JPA8001_18.

Tape, T.G. Interpreting diagnostic tests. The area under an ROC Curve. http://gim.unmc.edu/dxtests/ROC3.htm (accessed 23 September 2019).

Turner-Cobb, J.M., Koopman, C., Rabinowitz, J.D., Terr, A.I., Sephton, S.E., \& Spiegel, D., 2004. The interaction of social network size and stressful life events predict delayedtype hypersensitivity among women with metastatic breast cancer. International Journal of Psychophysiology, 54(3), 241-249. https://doi:10.1016/j.ijpsycho.2004.05.010.

US Department of Health., 1978. National Health Survey Disability Days United States 1975. Series 10. No 118. DHEW Publication No. (PHS) 78-1546. https://www.cdc.gov/nchs/data/series/sr_10/sr10_118.pdf (accessed 11 September 2019).

Watkins, M.W., 2000. Monte Carlo PCA for parallel analysis (computer software). https://download.cnet.com/Monte-Carlo-PCA-for-Parallel-Analysis/3000-2053_475332256.html (accessed 3 August 2019).

Weiland, T.J., Jelinek, G.A., Marck, C.H., Hadgkiss, E.J., Van Der Meer, D.M., Pereira, N. G., \& Taylor, K.L., 2015. Clinically significant fatigue: Prevalence and associated factors in an international sample of adults with multiple sclerosis recruited via the internet. PloS one, 10(2), e0115541. https://doi:10.1371/journal.pone.0115541

Zimet, G.D., Dahlem, N.W., Zimet, S.G., \& Farley, G.K., 1988. The Multidimensional Scale Of Perceived Social Support. Journal of Personality Assessment, 52(1), 30-41.

Zimet, G.D., Powell, S.S., Farley, G.K., Werkman, S., \& Berkoff, K.A., 1990. Psychometric characteristics of the Multidimentional Scale of Perceived Social Support. Journal of Personality Assessment, 55(3-4), 610-617. https://doi:10.1207/s15327752jpa5503\&4_17. 